\title{
Research on the Measurement of Classroom Teaching Quality Evaluation System Based on Entropy Weight TOPSIS Method
}

\author{
Jin $\mathrm{Liu}^{1, *}$ \\ ${ }^{1}$ College of Educational Sciences, Liaocheng University, Liaocheng, Shandong 252000, China \\ ${ }^{*}$ Corresponding author. Email: $1351298572 @ q q . c o m$
}

\begin{abstract}
Firstly, the importance of the construction of classroom teaching quality measurement system and the methods of classroom teaching quality measurement are summarized. This paper summarizes the domestic and foreign literature on the measurement of classroom quality evaluation system, further introduces the optimized measurement method of classroom teaching quality, and gives the logic of the index selection of classroom teaching quality. In the aspect of dimensionless processing, it focuses on the processing of incomparable and extreme values in the horizontal individual dimension and vertical time dimension. The two methods to determine the weight are given: one is entropy weight TOPSIS (Technique for Order Preference by Similarity to an Ideal Solution) method; the second is analytic hierarchy process. Finally, the specific steps of measuring the classroom teaching quality index system are given.
\end{abstract}

Keywords: The index system of classroom teaching quality, Range method, TOPSIS method of entropy weight, Analytic hierarchy process.

\section{INTRODUCTION}

Since the 1960s, Schultz and Becker's human capital theory holds that human capital was an important driving force for a country's long-term economic growth, the methods of the formation and accumulation of human capital include the following four aspects: first, education; second, training; third, health care; fourth, labor migration. Among them, education plays the most fundamental role in the formation of human capital, and the role of education in the formation of human capital is direct, effective and long-term. The logic of education to economic growth is that the ability of education to promote, the increase of human capital brought by the ability promotion will promote economic access. Under the reasonable economic and political system arrangement, economic growth will further promote the improvement of education level, which is a positive feedback spiral rise. Jin Jiuren believed that rural basic education was an important means to achieve targeted poverty alleviation, and analyzed the different connotations and realistic basis of "supporting rural education poverty" and "relying on education to support rural poverty", which was conducive to the implementation of the national "targeted poverty alleviation" strategy[1].

Education plays an important positive role in promoting economic growth and the strategy of "Rural Revitalization". Building a scientific and reasonable classroom teaching quality system has important guiding significance for accurate evaluation and scientific guidance of basic teaching. In 2018, at the national teaching conference, general secretary Xi Jinping stressed: "We must deepen the reform of the education system, improve the implementation mechanism of Lide Shuren, reverse the problem of unscientific teaching evaluation system, and fundamentally solve the problem of teaching evaluation baton." The construction of classroom teaching quality evaluation system is conducive to the development of education with socialist characteristics, further promoting the rapid growth of China's economy and rural revitalization, and realizing the Chinese dream of the great rejuvenation of the Chinese nation. 
The main body of teaching classroom quality measurement is students and teachers. The teaching ability of teachers and students' learning ability is related to the teaching quality of the classroom, among which teaching design, teaching practice and teachers' professional ability directly affect the learning effect of students. Most of the existing domestic literatures focus on the quality of classroom teaching by using subjective weighting method, which has a strong subjectivity. At the same time, they do not pay attention to the incompatibility of horizontal (individual) and vertical (time) dimensions. In this paper, combined with the relevant literature research at home and abroad, and with reference to the construction of other multi-dimensional index method, introduced the construction of teaching classroom quality measurement method. The marginal contribution of this paper is mainly reflected in the following two aspects: first, the entropy weight TOPSIS method and the coefficient of variation method + analytic hierarchy process method are introduced as the weight method to determine the composition of domestic classroom teaching quality indicators; Secondly, when constructing the classroom teaching quality index, this study focuses on the comparability of the horizontal (individual dimension) dimension and the vertical (time) dimension, and gives the solution, choosing the base period processing to ensure the consistency of the benchmark processing.

\section{INTRODUCTION TO THE MEASUREMENT OF THE EXISTING CLASSROOM TEACHING QUALITY EVALUATION SYSTEM}

The international research on the quality of classroom teaching began with the research done by Hiller, Fisher and kaess in 1969. Since then, the classroom teaching system has been in the process of continuous exploration and development. The FT teaching evaluation was developed in 1996 by a team led by Danielson and was recently updated in 2013.Class classroom interaction assessment system was developed by Piata team of the University of Virginia to assess the teaching quality from kindergarten to high school; The evaluation system started from three primary indicators to evaluate the teaching quality, which were emotional support, class management and education support. There were three to four secondary indicators under the three primary indicators, a total of 10 secondary indicators. Rtop classroom teaching quality model was formed in 1998, which is funded by the national foundation of the United States. It evaluated classroom teaching from teaching design and implementation, teaching content and classroom culture. MQI mathematics teaching quality evaluation system was developed by Hill team of University of Michigan. The first level index includes four dimensions: first, the richness of mathematical content; the second is how to deal with students' behavior; the third is error and imprecision; Fourth, students' participation in meaningful learning.

Most domestic researches focused on how to construct scientific teaching indicators to measure the quality of classroom teaching, Xie Dongxing took college physical education classroom teaching quality as the research object and took it as the key factor. He divided the teaching classroom quality into five first level indicators, namely teaching effect, teaching attitude, teaching content, teaching method and professional quality, and gave certain weight to these five indicators to measure the classroom teaching quality[2]. Tang Shunding used range method and hierarchical fuzzy evaluation method to evaluate the quality of teaching class[3]. Kang Ting used correspondence analysis method to analyze the key factors of classroom teaching quality, which was helpful for teachers with different characteristics to effectively improve the quality of teaching[4]. Kang Ting used correspondence analysis method to analyze the key factors of classroom teaching quality, which was helpful for teachers with different characteristics to effectively improve the quality of teaching[5].

Foreign mature teaching evaluation system is to comprehensively evaluate the quality of classroom teaching from multiple dimensions, and the first level index selection can cover all aspects of classroom teaching quality from different angles. There are some problems in the current research of classroom teaching quality rating system in China: First, the description of some sub dimension indicators is too broad, or it is difficult to observe and evaluate, and the operability is not strong. The same level of dimension index is disordered, resulting in the problem of repeated evaluation. Second, if each dimension index needs dimensionless processing, it is not found that the benchmark of each individual has changed in different years in domestic literature processing, which leads to the incompatibility of horizontal (individual) and vertical (time) dimensions, and leads to the construction of classroom teaching 
quality value cannot well reflect the teaching quality. Thirdly, few literatures introduce good objective weighting method to weight each dimension index.

\section{MEASUREMENT OF CLASSROOM TEACHING QUALITY RATING SYSTEM: LOGIC, SYSTEM AND METHOD}

\subsection{Measurement Logic and Index System}

Although the indicators of different dimensions of classroom teaching quality contain useful information of each dimension, if the indicators of a certain dimension are taken out as proxy variables of classroom quality, it may make a one-sided interpretation of classroom teaching quality and fail to comprehensively and accurately understand classroom teaching quality. Scholars need the evaluation system of classroom teaching quality evaluation should meet the following principles: first, the index must comprehensively and comprehensively reflect the quality of classroom education, and each dimension index of classroom teaching quality should scientifically reflect the overall perspective of classroom quality. Secondly, researchers should consider both horizontal (individual dimension) comparability and vertical (time dimension) comparability. Scholars usually say horizontal comparability refers to the comparison between different individuals in the same time dimension, and vertical comparability refers to the comparison of the same individual in different time dimensions. If scholars want to realize the comprehensive comparison of classroom quality, the constructed index must be able to achieve horizontal (individual dimension) and vertical (time dimension) comparability. Third, the evaluation system of classroom teaching quality must be scientific and reasonable, which can reflect the multi-level and diversification of classroom education quality. Based on the above principles and requirements, this article chooses the entropy weight TOPSIS method or analytic hierarchy process as the method of classroom teaching quality evaluation.

Using entropy weight TOPSIS method and analytic hierarchy process to evaluate classroom teaching quality needs to determine the first level index, second level index and third level index. Zhu Dequan and Song Naiqing used the method of factor decomposition to define the first and second level indicators in detail, and the first level indicators of the construction of classroom teaching quality index could be divided into teaching design, teaching implementation and teaching results [6]. Tan Dingliang and Zhu Jianming figured the evaluation index of junior high school mathematics teaching quality should be three dimensions: teaching design (teaching objectives, experimental content, experimental guidance and teaching links), teaching process (teaching behavior and learning behavior) and teaching effect (cognition, emotion and synthesis). At the same time, according to these three first level indexes, scholars continued to subdivide the second level indexes[7].

\subsubsection{Instructional Design}

Instructional design is a systematic arrangement of teaching activities before class, which reflects teachers' overall grasp of the classroom and plays a leading role in teaching quality. If there is no good teaching design, the knowledge absorbed by students in class will be fragmented and lack of logic, which will directly affect the quality of classroom teaching. The teaching design should also consider the students' acceptance ability and learning ability, and set goals according to the students' possible learning status tomorrow. Ye Lan and others believed that the teaching design of the first level indicators could set up three second level indicators according to different dimensions, which were the design of teaching objectives, the design of teaching content and the design of teaching process[8].

\subsubsection{Teaching Process}

After completing the teaching design, teachers need to teach according to students' learning ability, and fully cultivate students' comprehensive quality of this subject according to the needs of subject training. In the classroom, scholars should give full play to students' subjective initiative to achieve the goal of teaching and learning. Tan Dingliang and Zhu Jianming figured teaching implementation (first level indicators) should be divided into teaching behavior and learning behavior. Teaching behavior (second level indicators) includes whether classroom questioning was effective, guidance was appropriate, feedback was timely, generation goal was achieved, and attention was paid to abstract summary guidance; learning behavior (secondary indicators) included full participation of the whole process, efficient interaction and cooperation between teachers and students, hand brain 
cooperation, abstract generalization, expansion and transfer [7].

\subsubsection{Teaching Effect}

Tan Dingliang and Zhu Jianming believed that teaching achievements should include three secondary indicators: cognition, emotion and synthesis. Cognition included the following three dimensions: first, the understanding of knowledge and process; the second is the verification of principle and conclusion; the third is the discovery and solution of the problem. Emotion was divided into the following four dimensions: first, the enthusiasm to participate in the problem; Second, the curiosity of problem exploration; the third is the driving force of mathematics learning; fourth, selfconfidence in mathematics learning. The integration might include the following aspects: first, the transfer and expansion of knowledge and methods; second, the acquisition and development of students at different levels; third, the development tendency of teachers' ability [7]. Pang Lili figured the first level indicators of teaching effect should be divided into the following four secondary indicators through the dimension characteristics: first, knowledge and skills; second, language thinking; third, problem solving; fourth, emotional attitude [9].

\subsection{Exponential Dimensionless Treatment}

Before index synthesis, research must first make dimensionless treatment to the original indicators with different properties and measurement units, so as to ensure the additivity and comprehensive comparability of different dimensional indicators. The algorithm of range method for dimensionless processing of the original index is as follows:

$$
\mathrm{Y}_{i j}=\left\{\begin{array}{l}
\frac{X_{i j}-\min \left(X_{i j}\right)}{\max \left(X_{i j}\right)-\min \left(X_{i j}\right)}, \\
\frac{\max \left(X_{i j}\right)-X_{i j}}{\max \left(X_{i j}\right)-\min \left(X_{i j}\right)},
\end{array}\right.
$$

Among them, $X_{i j}$ is the $\mathrm{j}$ index of the first teacher, $Y_{i j}$ represents the standardized classroom quality index value after range method processing, min $\left(X_{i j}\right)$ represents the minimum value of $X_{i j}$. At the same time, considering the possible extreme value of each index, this paper shrinks the tail of the original index, which can avoid the extreme value and numerical distortion caused by various reasons. Because if each indicator is set by the upper and lower limits of each year's indicators, research will find that the benchmark of each individual changes in different years, so the longitudinal (time dimension) is not comparable. In order to compare the teaching quality of different teachers and different time, researchers need to select a benchmark year. Generally, scholars choose the starting year as the benchmark year. The dimensionless score range of the benchmark year processed by the above algorithm is between 0 and 1. The higher the score, the higher the teaching quality. The dimensionless values of the years after the base year may be less than 0 and greater than 1 . If there is no problem with the selected indicators and survey data, the absolute value of the dimensionless values obtained by the algorithm can accurately represent the level of teaching quality, and achieve the comparability of horizontal (individual) dimension and vertical (time) dimension.

\subsection{Weighting Method of Each Index}

After dimensionless treatment of each index, this paper needs to empower and add up the indicators. Through consulting a large number of literature, this paper summarizes two common methods of index empowerment, one is objective empowerment method, the main method is entropy weight TOPSIS method, entropy weight TOPSIS method is widely used to measure the quality of economic development, including papers of Weimin and Li Shuhao [10] and Liu Gefei and Ren Baoping [11]. TOPSIS method, which is used to measure economic quality, can also be used as a weighting method of classroom teaching quality. On the one hand, the entropy weight method is derived from the degree of variation of data, and has certain objectivity. On the other hand, TOPSIS is a simple and reasonable method to produce ranking results by comparing the relative distance between the scheme and the optimal solution and the worst solution. On the other hand, TOPSIS is a simple and reasonable method to produce ranking results by comparing the relative distance between the scheme and the optimal solution and the worst solution. The other is the subjective weighting method. The frequently used subjective weighting method is the analytic hierarchy process (AHP). Guo Feng et al. used the method of combining AHP with coefficient of variation to measure the digital inclusive financial index [12]. The author thinks that the combination of subjective and objective weighting method can also be used to measure the quality of classroom teaching. Next, researchers 
introduce the entropy weight TOPSIS method and analytic hierarchy process + coefficient of variation method.

\subsubsection{Entropy Weight TOPSIS Method}

Through formula (1), researchers standardize the data and get the matrix $\mathrm{Y}=\left(\mathrm{Y}_{\mathrm{ij}}\right)_{\mathrm{n} \times \mathrm{m}}$ Then, in order to avoid meaninglessness after taking logarithm, researchers shift the matrix:

$$
Y_{1}=\left(Y_{i j}+1\right)_{n \times m}=\left(a_{i j}\right)_{n \times m} \text { (2) }
$$

The information entropy of each index is calculated:

$$
E_{i j}=\ln \left(\frac{1}{n}\right) \times\left[\frac{a_{i j}}{\sum a_{i j}} \times \ln \left(\frac{a_{i j}}{\sum a_{i j}}\right)\right]
$$

Among them, $\mathrm{n}$ is the number of teachers. Then the weight of each index is:

$$
W_{j}=\frac{1-E_{j}}{\sum 1-E_{j}}
$$

In the third step, the weighted new matrix is obtained:

$$
Y_{2}=\left(W_{j} \times Y_{1_{i j}}\right)_{n \times m}
$$

Researchers define the elements in the new matrix as $d_{i j}$.

The fourth step is to use TOPSIS to calculate the best and worst solutions of each teacher's classroom teaching quality:

$$
Z_{j}^{+}=\left(\operatorname{maxd}_{i 1}, \operatorname{maxd}_{i 2}, \cdots, \operatorname{maxd} d_{i m}\right)
$$

$Z_{j}^{-}=\left(\operatorname{mind}_{i 1}, \operatorname{mind}_{i 2}, \cdots, \operatorname{mind}_{i m}\right)$

In the fifth step, the Euclidean distance between the optimal solution and the worst solution is calculated:

$$
\begin{aligned}
& D_{i}^{+}=\sqrt{\sum\left(Z_{j}^{+}-d_{i j}\right)^{2}} \\
& D_{i}^{-}=\sqrt{\sum\left(Z_{j}^{-}-d_{i j}\right)^{2}}
\end{aligned}
$$

The sixth step is to calculate the relative proximity of each measure:

$$
C_{i}=\frac{D_{i}^{-}}{D_{i}^{+}+D_{i}^{-}}
$$

Among them, $\mathrm{C}_{\mathrm{i}}$ is the relative closeness of each teacher's classroom teaching quality. It can be seen from formula (10) that $\mathrm{C}_{\mathrm{i}}$ is a value between 0 and 1. The larger the value is, the higher the quality of classroom teaching and the higher the score of teacher i will be.

\subsubsection{Analytic Hierarchy Process and Coefficient of Variation}

The combination of subjective weighting method and objective weighting method is coefficient of variation method + analytic hierarchy process. Guo Feng et al. used the method of combining AHP with coefficient of variation to measure the digital inclusive financial index [12]. Generally speaking, the use of analytic hierarchy process needs to be divided into different first level indicators through different dimensions. The first level indicators are divided into second level indicators through the diversity of indicators, and then the second level indicators are divided into specific indicators (third level indicators). Specifically, the method of coefficient of variation is used to calculate the weight of each specific index to the upper criterion layer, and then the total index is calculated by the weight of each criterion layer index to the upper target.

The basic idea of coefficient of variation method to determine the weight is the same as the entropy weight TOPSIS method, which determines the weight according to the variation degree of the dimensionless value of the original data. If the coefficient of variation of this index is particularly large, then the index has a greater explanatory power for the index, then it should be given a greater weight. The analytic hierarchy process (AHP) is a scientific and quantitative processing algorithm for solving qualitative problems. It is a comprehensive evaluation method for analysis and decision-making. Its main feature is to establish a hierarchical structure, simplify people's judgment into the comparison of the importance of two pairs of the most important variables of several variables, and transform the difficult qualitative comparison into operable quantitative judgment. Finally, the weight is determined and the final index is obtained by weight summation.

Whether entropy weight TOPSIS method or AHP + coefficient of variation method, it is widely used by scholars to construct some comprehensive 
indexes, especially the measurement of economic quality. Both methods have advantages and disadvantages.

The author thinks that the two weighting methods can be used to measure teachers' classroom teaching quality, and the Entropy TOPSIS method is more simple and objective. The author thinks that this method should be used as the preferred method to construct classroom teaching quality weighting, or both methods can be used as the measurement method to listen to experts' opinions, and choose the index that publishes the measure.

\section{SPECIFIC CONSTRUCTION STEPS OF CLASSROOM TEACHING QUALITY EVALUATION SYSTEM MEASUREMENT BASED ON ENTROPY WEIGHT TOPSIS METHOD}

The specific steps of the construction process of the classroom teaching quality index system are divided into three steps: first, the measurement index system is determined, including the determination of the first level index, the second level index and the specific index; second, according to the selected indicators, the measurement object and the survey object are determined, and the accurate data are obtained; third, the weight of each index is determined by using TOPSIS method or AHP + coefficient of variation method, and the scores of each index are further determined, and the total score of classroom teaching quality is obtained.

\subsection{Determining the Measurement Index of Teaching Classroom Quality}

Zhou Jiushi thank that the selection and construction of teaching classroom quality index system should follow three principles: First, we should systematically review the literature on classroom teaching quality at home and abroad, and fully explore the measurement logic of classroom teaching quality in the literature; The second is to repeatedly watch the classroom video of the teacher to be evaluated, and determine the evaluation index, and even need face-to-face communication with students and teachers. The third is to consult the opinions of education experts and famous teachers of the education system, and discuss with peers, so that experts and scholars can give clear feedback on the applicability of the overall framework of the evaluation system, the suitability and clarity of each dimension, and the clarity of descriptions of different dimensions [13]. In other words, it is roughly divided into two steps. The first step is to determine the primary selection target by referring to the literature, teaching video and the field investigation of the school. The screening measurement target should not only conform to the theoretical logic, but also be operable. If the operability is not strong, it is necessary to find alternative indicators, not only to determine the screening indicators, but also to determine the logic of the screening target, Provide theoretical logic for primary election objectives. The second step is the Delphi method to determine the indicators at all levels for further selection. That is to consult the relevant experts and scholars, design the questionnaire according to the primary goal of your screening, and set up an expert research team composed of education experts, famous teachers and researchers from the Ministry of education. Each expert makes further selection according to the measurement index and selection logic I initially selected, and determines the final level-1 index, level-2 index and specific measurement index through layer by layer screening.

\subsection{Preliminary Treatment of Indicators}

After the index is finally determined, the second step is to accurately get the data we need, and carry out dimensionless processing. Because a lot of data of classroom teaching quality indicators may be survey data, researchers must ensure that the investigators truthfully answer the questions on the survey data, because the authenticity and accuracy of the data is the key to the construction of classroom education quality index. To ensure the authenticity of the survey data, after obtaining the consent of the investigator, recording and video recording may be carried out, so as to ensure the authenticity of the survey data in the later stage. Two aspects should be paid attention to in the dimensionless processing of the survey data: one is to avoid the extreme data affecting the index value, and the article needs to reduce the tail of the data. The second is to ensure the horizontal (individual dimension) and vertical (time dimension) comparability of the data. At this time, the dimensionless processing needs to determine the base period, so as to ensure the consistency of the base period in different years. 


\subsection{Calculating the Weight of Measurement Index}

After the dimensionless processing of indicators, the next step is to determine the weight of measurement indicators. Considering the objectivity and easy operation, this paper recommends the entropy weight TOPSIS method. The algorithm has been described in detail in Chapter 2, and the specific steps are briefly introduced in this paper. First, the matrix is determined after dimensionless processing; second, to avoid the index logarithm, the matrix is shifted; third, the information entropy is determined, and the weight is further obtained. According to the index weight and dimensionless value, the value and new matrix are obtained. Fourth, the new matrix is obtained, and the optimal solution and the worst solution are calculated. Fifth, the Euclidean distance and relative proximity are calculated. After calculating the relative proximity, you can get the specific value of the index system, with the interval between 0 and 1. For clearer comparison, users can expand to 100 points according to the proportion, and the obtained value is the classroom quality index. The larger the index, the better the classroom teaching quality. At the same time, it can realize the horizontal (individual dimension) and vertical (time dimension) comparability. In this way, we can improve the teaching quality of different teachers and different time.

The evaluators can also measure the weight value and classroom teaching quality index through AHP method + coefficient of variation method, and compare the entropy weight TOPSIS method with the analytic hierarchy process method to see the difference between them and optimize the algorithm and index. The calculation and optimization of indicators need constant exploration. After knowing the measurement system and values, teachers can make up for their own teaching shortcomings and improve the quality of classroom teaching according to the measurement theory and specific indicators. At the same time, we should continue to explore more accurate measurement system.

\section{CONCLUSION}

This paper gives the logic of the index selection of classroom teaching quality, describes the classroom teaching quality through the first level index of three dimensions of teaching involvement, teaching process and teaching effect, and gives the specific measurement method. Secondly, the dimensionless processing of each specific index is carried out. In the aspect of dimensionless processing, it focuses on the processing of the incomparable and extreme values of the horizontal (individual) dimension and vertical (time) dimension, and gives two methods to determine the weight: one is the entropy weight TOPSIS method; the other is the entropy weight TOPSIS method; the second is analytic hierarchy process. After that, the specific steps of measuring the classroom teaching quality index system are given. After obtaining the specific classroom teaching quality, the government and management can evaluate the teaching quality of teachers through qualitative and quantitative methods. Teachers can make up for their teaching shortcomings and improve the quality of classroom teaching according to the measurement theory and specific index values. At the same time, this paper is helpful to the scholars to explore more accurate measurement system.

\section{AUTHORS' CONTRIBUTIONS}

This paper is independently completed by Jin Liu.

\section{REFERENCES}

[1] Jin Jiuren. Connotation of poverty alleviation through education and path transformation $[\mathrm{J}]$. Education and economy,2020,36(02):10-18.

[2] Xie Dongxing. Reconstruction of classroom teaching quality evaluation system in Colleges and universities $[\mathrm{J}]$. Journal of Shandong Institute of physical education, 2016,32 (01): 105-113.

[3] Tang Shunding, Meng Qiong, Song Ying, Bai Hao, Deng Li, Zhang Xiaoqing. Comparison of fuzzy comprehensive evaluation method and extreme difference method in classroom teaching quality evaluation in Colleges and universities [J]. Journal of Kunming Medical University, 2020,41 (03): 154-159

[4] Kang Ting. Application of correspondence analysis in classroom teaching quality evaluation $[\mathrm{J}]$. Practice and understanding of mathematics, 2015,45 (01): 80-87

[5] Wang Yane, Liang Yan, Ding Xin'an. Study on the evaluation standard of classroom teaching quality based on cluster analysis [j]. Software introduction, 2021,20 (02): 234-237. 
[6] Zhu Dequan, Song Naiqing. Educational statistics and evaluation technology [M]. Chongqing: Southwest Normal University Press, 2007:376-395

[7] Tan Dingliang, Zhu Jianming. Teaching evaluation of junior high school mathematics experiment $[\mathrm{J}]$. Curriculum. Teaching materials. Teaching methods, 2016,36 (08): 108-113

[8] Ye Lan, Wu Yaping. Reform of classroom teaching and classroom teaching evaluation reform: theoretical and practical exploration of classroom teaching reform in new basic education [J]. Education research, 2003 (8): 42-49.

[9] Pang Lili. Research on "student oriented" classroom teaching evaluation standard [D]. Wuhan: Huazhong University of science and technology, 2007.

[10] Wei Min, Li Shuhao. Research on the measurement of China's high quality economic development level in the new era [J]. Quantitative economic and technological economic research, 2018,35 (11): 3-20

[11] Liu Gefei, Ren Baoping. Path selection of cultivating new kinetic energy for high quality development of local economy [J]. Financial science, 2020 (05): 52-64.

[12] Guo Feng, Wang Jingyi, Wang Fang, Kong Tao, Zhang Xun, Cheng Zhiyun. Measuring the development of digital Inclusive Finance in China: index compilation and spatial characteristics $[\mathrm{J}]$. Economics (quarterly), 2020,19 (04): 1401-1418.

[13] Zhou Jiushi. Analysis on teaching quality of high quality mathematics courses in middle schools [D]. Shanghai: East China Normal University, 2018. 